\title{
Los zorros: lenguaje, realismo y política en la novela póstuma de José María Arguedas*
}

\author{
Los zorros: language, realism, and politics in the \\ posthumous novel by José María Arguedas
}

Juan Camilo Lee Penagos**

\begin{abstract}
RESUMEN
El artículo hace un análisis de El zorro de arriba y el zorro de abajo, novela póstuma de José María Arguedas, en donde se relacionan el contexto político peruano y latinoamericano, la trayectoria del autor y las discusiones al interior de los campos literarios del país y la región. Se pone especial atención a la concepción de la relación entre lenguaje y realidad que plantea la novela, y la manera en que esto se relaciona con las posturas políticas de Arguedas. Antes de analizar la novela, el artículo presenta los debates en los que participó el autor un par de años antes de la escritura de la novela y la manera en que estos se verificaban en torno a los conceptos de "realismo" y "vanguardia" en Latinoamérica y el Perú.
\end{abstract}

\begin{abstract}
This article will analyze "El zorro de arriba y el zorro de abajo," José María Arguedas posthumous novel. We will attempt to link it to Perú and Latin America's political context, the author's trajectory, and discussions inside the country and region's literary fields. Our focus will surround the relation between reality and language that the novel proposes and how it relates to Arguedas' political stances. Before analyzing the novel, we will present, as
\end{abstract}

Palabras clave:

José María

Arguedas, realismo, Los zorros, literatura peruana, indigenismo, literatura latinoamericana.
Keywords: José María Arguedas, Realism, Los zorros, Peruvian Literature, Indigenism, Latin-American Literature.

\footnotetext{
* El artículo es resultado parcial y con algunas modificaciones de mi trabajo de investigación doctoral "La emergencia de lo real. Creación y política en los '60": obras, textos y autores latinoamericanos", financiado por Colciencias y la Universidad Nacional de Colombia.

** Colombiano. Doctor en Ciencias Humanas y Sociales de la Universidad Nacional de Colombia, sede Medellín. En la actualidad desarrolla un proyecto de investigación postdoctoral en la Facultad de Artes de la Pontificia Universidad Javeriana en Bogotá, Colombia. juancamilolee@gmail.com
} 
Los zorros: lenguaje, realismo y política en la novela póstuma de José María Arguedas | Lee

contextualization, Arguedas' debate about "realism" and "avantgarde" in Latin-America and Peru years before the writing of "Los zorros." 


\section{Introducción}

El objetivo de este artículo es modesto: pretende, desde una mirada construida a partir de la teoría de los campos de Pierre Bourdieu, encontrar las posiciones políticas de Arguedas que se expresan, explícita o implícitamente, en su obra El zorro de arriba y el zorro de abajo (1971) (en adelante Los zorros), relacionarlas con las propuestas estéticas que expone la novela, y poner de manifiesto la manera en que estas dos cuestiones se tejen y tensionan en las discusiones del campo literario peruano y latinoamericano.

Digo modesto porque la complejidad de la novela ha sido discutida por diversos pensadores y pensadoras, desde los más diversos puntos de vista, evidenciando una riqueza que no aspiro a abarcar, ni de cerca, en el presente artículo. En las discusiones contemporáneas en torno a la literatura latinoamericana cabe destacar el libro de Mabel Moraña (2013) acerca de Arguedas y Vargas Llosa, donde se expone la diferencia radical entre los dos intelectuales, y la relevancia de esta oposición en el contexto actual de neoliberalismo.

En este sentido, aunque con algunos años de diferencia, cabe recordar también el papel de la obra de Arguedas en las discusiones referidas a la mundialización y el mercado internacional de la literatura, aguzadas por la propuesta de Pascale Casanova y revisadas por diversos autores en América Latina en la "literatura mundial" editada por la Universidad de Pittsburg de la mano de Ignacio Sánchez Prado (2006). En este libro, en los capítulos escritos por Mabel Moraña y por Jean Franco, Arguedas aparece justamente como el autor insigne cuyo ejemplo hace tambalear los cimientos de la "república mundial de las letras". Por supuesto, las ya clásicas lecturas de Ángel Rama , Antonio Cornejo Polar, Martín Lienhard, Sara Castro-Klarén, William Rowe, entre otros, sientan las bases para las discusiones en torno a la obra de Arguedas, teniendo en cuenta las complejidades culturales que plantea. Sin embargo, habida cuenta de la importancia de estas lecturas, este artículo se propone señalar un aspecto que, al parecer, ha sido dejado de lado por quienes han pensado la obra de Arguedas, en especial Los zorros: su ubicación específica en la coyuntura política peruana y latinoamericana de finales de los años 60 .

Para esto, la mirada bourdiana es privilegiada. La propuesta de los "campos" de este sociólogo francés facilita la comprensión de las rela- 
ciones entre las posturas estéticas de los autores, sus posiciones en los debates de cada campo o disciplina, y las implicaciones políticas de cada una de ellas. Es especialmente valiosa en la propuesta de Bourdieu (1995) la concepción de las posturas estéticas - y de la diferenciación entre ellas-, como parte de las contiendas por la legitimidad y el "capital específico" de cada campo: no hay detrás de estas, en general, concepciones sólidas o positivamente identificables, sino que mucho dependen de las necesidades de los agentes en aras de legitimarse, diferenciándose de sus contendores y dependiendo de su trayectoria y su "posición" dentro del campo de fuerzas de su labor (188).

De esta forma, las tensiones y conflictividades de la generalidad social se experimentan y expresan de maneras específicas, pero "estructuralmente homólogas" al interior de los campos que poseen por esto una "autonomía relativa": tienen sus propios códigos y formas de legitimación independientes de otros campos, pero a la vez dependen y expresan conflictos sociales y políticos de mayor envergadura a través de esos códigos (Bourdieu, "El campo..." 15-18).

Por otra parte, desde una mirada latinoamericana, una de las críticas que se han hecho a la teoría bourdiana es la excesiva atención a la dinámica nacional que se le presta para comprender a los agentes culturales, puesto, que como es sabido, en el continente muchas de las maneras de legitimación en estos campos son regionales o internacionales, y esto complejiza el análisis de la "autonomía" de los campos latinoamericanos (Martínez, "Para estudiar..." 2007; Sigal 105-107). Teniendo en cuenta lo anterior, el artículo pretende ubicar las posturas estéticas de Arguedas y sus intervenciones en el campo literario, tanto en las discusiones nacionales peruanas como en las regionales latinoamericanas, mostrando la manera en que el autor entiende su trayectoria y su posición en el campo, y la forma en que Los zorros interviene en esas discusiones.

El artículo rastrea, en primer lugar, las posturas de Arguedas en las discusiones, para luego ubicar esas ideas en la novela y entender cómo se complejizan en la obra. También muestra cómo la novela se inserta en las discusiones de los campos y reactualiza la trayectoria de Arguedas en la nueva configuración del campo literario latinoamericano después de 1968. Por último, el artículo perfila algunas de las implicaciones políticas de la novela tanto en Perú como en Latinoamérica 
y del suicidio del autor. Como se indicaba anteriormente, el artículo se concentra en lo mencionado en estas líneas, sin desconocer que la novela de Arguedas posee una complejidad que está muy lejos de agotarse con esta aproximación.

\section{Arguedas y los debates}

En 1965 se realizó el Primer Encuentro de Narradores Peruanos, donde participaron varios de los más reconocidos escritores del Perú, como Ciro Alegría, Mario Vargas Llosa, Sebastián Salazar Bondy y Arguedas, entre otros. Allí se discutieron diversos temas de interés para el campo literario del momento, y Arguedas hizo explícitas algunas de sus posturas respecto de la labor literaria y la realidad social y política del país. En particular, la relación entre literatura, realidad y sensibilidad serrana fue el tema recurrente en las intervenciones de Arguedas, quien entabló un amistoso debate con Salazar Bondy. Arguedas reforzaba su postura en cuanto a la necesidad de una literatura que tuviera un contacto real y vivencial con el Perú, haciendo énfasis en la relación del ser humano con la naturaleza y en la forma en que la cultura quechua privilegiaba ese vínculo (Obra... 7, 114). La polémica surgió porque Salazar Bondy planteaba que la literatura era una mentira, "la más maravillosa de las mentiras" (112). Arguedas, descontento con esta definición, insistía en que su obra era radicalmente verdadera, real. Es pertinente anotar que él, en aras de reforzar su argumento, planteaba que la literatura argentina en general carecía de un contacto "real" con su situación social concreta, y que se basaba en literaturas extranjeras. También habría que remarcar que la concepción de Arguedas acerca de lo "real" en la literatura estaba directamente relacionada con su idea del lenguaje: "La palabra es nombre de cosas o pensamientos o de reflexiones que provienen de las cosas, de lo que se piensa sobre las cosas; lo que es realidad verbal es realidad realidad [sic] [...] yo no acepto la realidad verbal, las palabras nombran cosas" (Obra... 7, 132). De esta manera, Arguedas ratifica que el lenguaje que él propone tiene una relación ontológica con el mundo: "si hay una realidad verbal, debemos entender que se trata de una realidad objetiva" (136).

Continuando con esta noción de la literatura como expresión de un contacto directo con la realidad, Arguedas plantea que existe una 
diferencia notable entre una literatura creada desde la inocencia y la intuición, y aquella creada a partir del conocimiento consciente de tradiciones y técnicas narrativas. Por supuesto afirma que él escribe desde la vida misma, desde el desconocimiento de las técnicas y que otros autores, como Mario Vargas Llosa, lo hacen desde el desarrollo de ellas (Obra... 7, 156). Esta cuestión se torna más interesante en cuanto algunos participantes del debate (Alegría y Salazar Bondy) plantearon la cuestión de si la técnica literaria consistía en un conjunto de influencias foráneas, incluso si constituía un caso de colonialismo cultural, y si era necesario que la literatura latinoamericana construya una técnica propia (164-165). Aunque Arguedas no participó o intervino directamente en este tema, es necesario llamar la atención sobre el hecho de que se planteara la cuestión en estos términos, pues ofrece una muestra respecto de los intereses del campo literario peruano y latinoamericano. El hecho de que se construyera, en esta discusión, una especie de dicotomía entre los escritores "espontáneos" y los "técnicos", y que el manejo de la técnica se asimilara a autores más "internacionales" mientras la ingenuidad lo hiciera a autores más "regionales", estaría anticipando uno de los debates más sonados entre Arguedas y Julio Cortázar, que se daría un par de años después. Esta división sería de primordial importancia en la configuración del campo literario tanto a nivel nacional en el Perú como a nivel regional en Latinoamérica. Arguedas defiende en este debate su trayectoria como autor "indigenista" consagrado a nivel nacional, que se opone a la literatura que pretende insertarse en el mercado internacional del libro, usando técnicas narrativas sofisticadas, representada muy bien por su compatriota Vargas Llosa.

Ahora bien, este debate puede entenderse en el Perú en esos años como una cuestión entre los nuevos narradores como Vargas Llosa y autores ya consagrados como Arguedas, mientras que, a nivel político, se percibe como una forma de señalar la necesidad de una organización política por parte de los indios serranos (Lee, "Política en..." 24). Como se decía, estas pugnas responden a una necesidad de los agentes de diferenciarse unos de otros, más que de una intención de definir características concretas de las obras o sus posiciones. En este sentido, las definiciones conceptuales acerca del "realismo" o "vanguardia" no son lo que realmente se discute entre los agentes, sino que estos signi- 
ficantes reúnen ciertas características de las obras, cierto tipo de trayectorias y posición política con límites fluctuantes que responden a los intereses de los agentes y a la estructura del campo en determinada coyuntura, es decir, no funcionan como términos claramente determinados, sino como significantes diferenciadores (Bourdieu, Las reglas del arte... 188).

Por otra parte, en 1965 se realizó la Segunda Mesa Redonda sobre Literatura Peruana y Sociología, al alero del Instituto de Estudios Peruanos, en donde se reunieron escritores y destacados sociólogos para conversar sobre Todas las sangres (1965). Esta reunión ha pasado a la historia de la literatura peruana como un desafortunado encuentro entre los sociólogos y Arguedas, e incluso se ha dicho que fue uno de los episodios centrales en la crisis sicológica del escritor, la que terminaría con su suicidio en 1969. Lo cierto es que en este encuentro se evidenciaron las profundas diferencias entre los planteamientos implícitos en Todas las sangres y el enfoque de los sociólogos que asistieron. La crítica principal que se le hizo a la novela desde la mirada de los sociólogos asistentes fue que no se trataba de "un testimonio válido para la sociología" (Arguedas et al., ¿ He vivido... 30). Si se piensa en la noción de Arguedas acerca de la necesidad de que la literatura naciera de un conocimiento profundo de la realidad del país, tal juicio de su novela era devastador. Sin embargo, en buena medida, a la novela se le criticaba que no reafirmara clara y explícitamente ciertas concepciones de cuño marxista que imperaban en el campo de las ciencias sociales del momento en el Perú (Moore 89). Tal y como Arguedas ratifica en sus intervenciones en ese debate, Todas las sangres plantea la posibilidad de un aporte político del mundo serrano al mundo contemporáneo a través de una sensibilidad de conexión con el mundo natural que está basada en relaciones de cooperación y trabajo comunitario. El hecho de que este planteo no se amoldara a las teorías racionalistas marxistas hizo que Bravo Bresani lo calificara como error (Arguedas et al., ¿ He vivido... 42). En este sentido, Arguedas se aleja de los postulados marxistas a nivel político, planteando con el personaje de Rendón Wilka una versión mágica y serrana de rebelión, que toma su fuerza emancipatoria de las características culturales de las comunidades indias. 


\section{Realismo, Arguedas y Latinoamérica}

A principios de 1968 Arguedas fue convocado por la Casa de las Américas para ser jurado de uno de los premios en literatura. Según Claudia Gilman (2003), la pregunta por el "realismo" atravesó las discusiones que se dieron durante toda la década de los 60 en el campo literario latinoamericano, y se desarrolló paralelamente a la pregunta por su relación con la "vanguardia". Como lo indica esta autora, en el armazón crítico que elevó la novela como el género literario privilegiado en Latinoamérica durante esa década, se buscó una suerte de equilibrio entre las búsquedas de un nuevo realismo que superara la condición prescriptiva del realismo socialista y que, al tiempo, pudiera nutrirse de las innovaciones técnicas de tradiciones literarias foráneas. Esta relación se declaró como posible hasta el año de 1968, cuando según Gilman partió en dos la historia del campo literario que se forjaba en Latinoamérica. De allí en adelante, la cuestión de la posibilidad vanguardista como uso novedoso de técnicas se fue convirtiendo en algo casi contrarrevolucionario, y al contrario de lo que había sucedido hasta 1967, no podría ser este término más que el polo opuesto del "realismo" (317-323).

En este escenario, resulta curioso que un autor como Arguedas haya sido convocado para el año en que dicha transformación se estaba produciendo. Como se mencionó, Arguedas se consideraba a sí mismo como un escritor "realista" que prescindía de las técnicas novedosas y foráneas que renovaban la literatura en el Perú y en Latinoamérica, y era estimado, según se veía en el debate aquel encuentro de escritores, de la misma manera por varios autores reconocidos de su país. Todo esto trajo como resultado que después de 1968 Arguedas quedara del lado de los "realistas" — opuesto a los "vanguardistas" en las nuevas condiciones del campo literario.

Este cambio en la manera de entender el género novelístico fue de crucial importancia, puesto que varios de los grandes autores que habían forjado su trayectoria en el ámbito generado por la Casa de las Américas y la Revolución cubana eran novelistas consagrados, y que en buena medida se podían considerar como "realistas de vanguardia". Es así que a nivel latinoamericano, antes de 1968, esta dualidad descrita no era tajante; el campo literario no se había dividido de la manera 
en que lo haría luego de la muerte del Che Guevara y el "caso Padilla" . En este sentido, la radicalización del campo político latinoamericano debido a las presiones internacionales sobre el gobierno revolucionario cubano se "refracta" en el campo literario, dando como resultado una división tajante entre los "realistas" y los "vanguardistas", lo cual redefine los debates específicos del campo, las características que se agruparían bajo cada término, y las implicaciones políticas para cada uno de ellos.

En efecto, después de 1968, cuando esta pareja de términos dejó de ser posible, se empezó a construir un discurso que impugnaba fuertemente no solo las relaciones entre "realismo" y "vanguardia", sino todo el sistema crítico que había avalado a tales autores: "La generalización del discurso antiintelectualista puso en cuestión la agenda cultural (por otra parte ya realizada) y no solamente el experimentalismo o el impulso hacia lo nuevo. Se atacó in toto a la nueva narrativa latinoamericana" (Gilman 332). Es decir, las nuevas condiciones políticas mundiales y la manera en que afectaban las políticas culturales de Cuba -y por consiguiente los debates de la intelectualidad crítica latinoamericana-, convertían a quienes habían sido hasta el momento autores insignes de la cultura revolucionaria en blanco de críticas. Este nuevo estado del debate literario en Latinoamérica se manifestaría de manera particular en la polémica de 1969 entre Arguedas y Cortázar, haciendo eco cada uno de la dicotomía entre el escritor "realista" y el escritor "vanguardista”, el "cosmopolita” y el "provinciano", el "exiliado" y el "que se quedó".

\section{Los zorros y el lenguaje sin realidad}

La novela Los zorros fue publicada en 1971, dos años después de que Arguedas se suicidara en 1969. En la novela se pueden encontrar tres tipos de registros narrativos: el relato de la lucha de una voz narrativa,

1 El "caso Padilla" refiere a lo sucedido con el poeta cubano Heriberto Padilla, en el año 1971, cuando fue arrestado y privado de su libertad durante más de un mes, y luego obligado a leer públicamente una "autocrítica", donde se arrepentía de sus escritos. Esto se dio por la lectura de algunos poemas que fueron interpretados como antirrevolucionarios. Padilla había sido ganador, en 1968, del concurso de poesía de la Casa de las Américas, con José Lezama Lima como jurado. Este "caso" marcó un parteaguas en la historia de las relaciones entre intelectuales latinoamericanos y la dirección política revolucionaria en Cuba. 
en forma de "diarios", contra del impulso de la muerte, y en donde se asume la escritura como arma vital en esa contienda; los capítulos o "hervores" en torno a la vida de algunos personajes en la ciudad costera e industrial de Chimbote; $y$ las pocas y significativas intervenciones de "el zorro de arriba" y de "el zorro de abajo", personajes míticos a los que Arguedas les da voz en la novela. Dentro de los diarios se encuentran reflexiones acerca del proceso creativo de la misma novela - Los zorros sería una novela "autoconsciente" - , de la vida personal del narrador, su trayectoria literaria, y la conformación del campo literario latinoamericano. En las acciones que ocurren en Chimbote, en tanto, aparece una serie de personajes, varios de ellos provenientes de la sierra, que intentan sobrevivir en medio del caos y la sordidez del ambiente. Hay en estos fragmentos un fuerte interés por mostrar las características del lenguaje utilizado por los personajes. Por otro lado, en apariencia, la novela está inconclusa: dentro de los mismos diarios el narrador se confiesa vencido por la muerte, y nombra los capítulos que pensaba escribir si hubiera podido soportar un poco más la vida. Las intervenciones de "los zorros", aunque pocas y cortas, son significativas en cuanto a que le dan el nombre a la obra, y porque refieren al mundo mítico serrano. "Los zorros" son personajes provenientes de relatos indios, con toda una significación cosmológica, y sus intervenciones apuntan a enmarcar tanto la vida de Arguedas como la narración en Chimbote dentro de márgenes temporales y espaciales de carácter mitológico. Es, como se ve, una novela de gran complejidad estructural y narrativa: no sería descabellado pensarla como la novela más "vanguardista" de Arguedas, sin dejar de tener un fuerte contenido "realista" 2

La novela desde el principio pone como tema privilegiado el de la relación del lenguaje con la realidad - y para Cornejo (1973) con el impulso vital (Los universos... 309) —, no solo a través del complicado lenguaje que aparece en el primer capítulo de la narración en Chimbote, que es prácticamente incomprensible para los lectores, pero que constituye el habla cotidiana de los pesqueros de la zona. También esta

2 Cabe insistir en que estas caracterizaciones no apuntan a definir la novela conceptualmente, sino más bien a inscribirla dentro de la terminología de los debates contemporáneos, dando cuenta de las tensiones específicas del campo literario que la novela expresa, sin pretender con esos rótulos una comprensión académica o teórica. 
relación aparece en las reflexiones de los diarios acerca de la construcción misma de la novela y de cómo esta afecta los intentos del narrador por sobreponerse al impulso de muerte que vibra fuertemente en su siquis:

El encuentro con aquella alegre mujer [una zamba prostituta] debió ser el toque sutil, complejísimo que mi cuerpo y alma necesitaban, para recuperar el roto vínculo con todas las cosas. Cuando ese vínculo se hacía intenso podía transmitir a las palabras la materia de las cosas ${ }^{3}$. (El zorro... 7)

Esto aparece en las primeras líneas del "Primer diario" y el tema se convertirá en uno de los principales en la novela, cobrando diferentes sentidos y matices. Esta incapacidad confesada por la voz de los diarios -que es muy tentador llamar directamente Arguedas, pero que resultaría un error- se ve reflejada en los capítulos respecto de Chimbote, por cuanto una de las características más destacadas de las obras de Arguedas está ausente: no se encuentran momentos de comunicación íntima con el mundo que se constituyen en las características que Arguedas propone como la fuerza política que reside en la sensibilidad serrana (Lee 23-25), esos momentos de magia casi que han dejado de existir y han sido reemplazados por momentos en que algún canto o habla de los personajes transmite la vida en Chimbote, pero no la vida o el mundo como totalidades, como en las novelas anteriores. Tal vez en el habla de los zorros o en el canto del personaje ciego se encontraría algo de esa magia, pero su presencia dista de ser tan constante como en las obras anteriores y, de hecho, pareciera que estas someras apariciones estuvieran allí para contrastar con el lenguaje que domina el resto de las situaciones y los personajes.

En otro artículo dije que el proceso social que más interesaba a Arguedas para ser representado literariamente era el de las migraciones indias hacia la costa (Lee 12-13). Se ha visto cómo la necesidad de transmitir un vínculo "material" con el mundo peruano se había constituido para Arguedas en el punto crucial sobre el cuál construir una obra literaria de valor. Así, Los zorros se erige como una contradicción muy fuerte dentro de la trayectoria del autor: abarca el tema

3 Cursivas del autor. 
que este venía planteando desde hacía más de una década como primordial para la literatura peruana de la época, pero lo hace desde la confesión de la incapacidad de ese contacto "material" con las cosas, característico del lenguaje literario que él manejaba en sus anteriores obras. Por otro lado, hay también una clara intención de Arguedas de salirse de las convenciones del género narrativo que venía manejando: Los zorros es una novela que, debido a sus múltiples registros narrativos, y sobre todo al uso novelado de lo que serían diarios personales del autor, abandona esa "espontaneidad" o "ingenuidad" de la que se enorgullecía en el Primer Encuentro de Escritores Peruanos de 1965, y pareciera acercarse a una forma más intelectualmente elaborada de construcción de las estructuras narrativas, de alguna manera acercándose al polo "vanguardista" de la dualidad realismo-vanguardia que se estaba construyendo en los debates literarios de la intelectualidad cubana y latinoamericana de izquierda en 1968.

Como se mencionaba, el polo del "realismo" —que antes de 1967 no se oponía radicalmente al polo "vanguardia" -, empezó a considerarse privilegiado en las discusiones literarias que se producían alrededor de la Casa de las Américas. Tal contraposición se podía encontrar ya cifrada para el Perú en el amistoso debate entre Salazar Bondy, Alegría y Arguedas en el encuentro de escritores de 1965, en donde los dos últimos se declaraban ajenos a la técnica narrativa y su creación era algo así como "espontánea". También en 1969 en la polémica nada amistosa- con Cortázar, se podría rastrear esta misma polaridad. Lo que polemizaban Arguedas y el autor de Rayuela era, según Mabel Moraña (2010), "la posición de ambos escritores en torno a la relación entre lo nacional y lo foráneo o, más precisamente, entre la labor intelectual desplegada in situ, dentro del territorio nacional, y la desarrollada en el exterior" (149). Como se veía en el debate de 1965, lo foráneo en la literatura se asociaba al manejo de las técnicas, mientras lo local se relacionaba con la espontaneidad. Así, la línea que Arguedas venía defendiendo como autor "realista" y cuya experiencia se afincaba necesariamente en la relación material con el mundo andino que se retrataba en sus obras, de alguna manera se resignificaba en el contexto ya más radicalizado de la Casa de las Américas después de 1968, lo que se hacía explícito en la polémica con Cortázar: Arguedas defendía, en términos bourdianos, su capital cultural acumulado hasta entonces a través de una posición "realista" o "indigenista". Sin embargo, si en 
1965 se podía discutir amistosamente acerca de la técnica narrativa y su componente foráneo, en 1968 el tema pasaría a lo personal y sería una polémica álgida y ofensiva. Su posición "provinciana" le haría frente al "cosmopolitismo", y haría eco de las críticas a la nueva narrativa latinoamericana que se empezaban a gestar en Casa de las Américas desde 1968. Sin embargo, sigue existiendo al respecto una contradicción: al tiempo que su postura "realista" y de contacto con el mundo peruano se reafirmaba a través de su "provincialismo", la novela Los zorros abandona el "espontaneísmo", es decir, habría una aparente contradicción entre el plano de la "posición" en el campo (Arguedas como autor consagrado en el polo ahora legitimado del "realismo"), y el de las "tomas de posición" (Los zorros como "toma de posición" que puede entenderse como "vanguardista"). Pero antes de observar esta contradicción, cabe detenerse en la manera en que la novela se convierte en una crítica a la corriente "vanguardista" y cosmopolita del campo literario latinoamericano.

Alberto Moreiras (1997) muestra en su artículo cómo la obra pone en crisis - de una vez por todas- el "realismo mágico" latinoamericano y la propuesta de "transculturación" de Ángel Rama. Dicha investigación relaciona la reflexión de la novela en torno al lenguaje y la realidad, y el debate acerca de la cuestión de las culturas indígenas en Latinoamérica. El argumento principal de Moreiras, grosso modo, es que Los zorros muestra la imposibilidad de llevar a cabo una verdadera "transculturación", mostrando finalmente que detrás del proyecto de Ángel Rama y la propuesta del "realismo mágico" se halla una intención de subordinar las culturas no occidentales al proyecto de modernización eurocentrista (101). Si bien Moreiras analiza la novela desde una perspectiva descolonial, y hace una reflexión que tiende más a lo teórico que a lo histórico, establece ciertas ideas que parecen primordiales para el análisis de Los zorros. Interesa aquí principalmente que Moreiras plantea la novela como un "passionate signifier of the end of signification" (103) o asegura que "meaning, in this novel, results from meaning's absolute implosion” (104), y que lo haga para mostrar la imposibilidad de la transculturación. La aporía que aparece así en Los zorros refiere no solamente al hecho de que Arguedas haya elaborado una novela mediante un uso del lenguaje radicalmente distinto del que había utilizado hasta entonces, esto es, un uso ontológicamente distinto, sino también de que lo haya hecho en el momento histórico preciso 
cuando defendía su posición como autor "realista" y, sobre todo, como autor in situ, es decir, con un contacto "material" con su patria: aparentemente Arguedas escribía una novela que, en cierta medida, iba en el sentido contrario de su anterior trayectoria, y que estaría contradiciendo su "posición" en el campo literario latinoamericano.

Se propone detenerse en esas dos interesantes expresiones de Moreiras, y desarrollar lo que plantean: "La novela como apasionado significante del fin de la significación" y "El significado, en esta novela, resulta de la implosión absoluta del significado". Ambas expresiones apuntan al hecho de que en la novela aparece el significante, en buena medida, desligado de su significado: es una novela acerca del lenguaje que se separa de su función denotativa y esta separación es narrada tanto en los diarios - como búsqueda infructuosa por parte de la voz del diarista de recuperar su capacidad para escribir y para volver a imprimir a las palabras la materia del mundo- como en los "hervores", en forma del uso "babélico" del lenguaje por parte de los personajes. Por ejemplo, es notable que el primer capítulo muestre el lenguaje vulgar y casi inentendible de un pescador, mientras el último termine con una cita de la "Carta de San Pablo a los Corintios" en donde se reflexiona acerca de la capacidad del lenguaje y el conocimiento para acercarse a la divinidad: "Si yo hablo en lenguas de hombres y de ángeles [...]", y el personaje - un sacerdote activo en Chimbote- que lo está leyendo piensa para sí mismo: “¿Para qué tanta inteligencia y tanto estudio? ¿Es malo decir concha'e tu madre, Señor? ¡No, no es malo! Procura ardencias necesarias en las neuronas y en la sangre" (Arguedas, El zorro... 241). También los zorros, en una de sus intervenciones, mencionan el asunto: "El zorro de arriba: La palabra es más precisa y puede confundir. El canto de pato de altura nos hace entender todo el ánimo del mundo" (49).

Para continuar con este análisis, se propone un ejercicio: suponer por ahora que el autor de carne y hueso Arguedas no se suicidó, y que todos los diarios y reflexiones sobre la muerte son ficciones que hacen parte de una novela en donde el autor se coloca a sí mismo como personaje. Esto no en aras de hacer un análisis del texto "en sí mismo", sino para comprender mejor la relación del texto con las circunstancias que lo hicieron posible. La cuestión de la muerte dentro de la novela es compleja, incluso sin tener en cuenta que Arguedas 
se suicidó en "realidad". Así, al suponer que el suicidio es una ficción al interior de la novela, se podría decir que la obra está completa, es decir, que su incompletitud es parte de lo que ella enuncia sobre sí misma - dentro de su propia ficción- lo cual es parte integral de su sentido: la muerte de su autor ficticio no sería una interrupción verdadera. La novela narraría su propio proceso de escritura como una interrupción, y esta última se constituiría como parte de su propio significado. Ahora, ¿ cómo es ese autor ficticio que la novela fabrica para luego darlo por muerto y ella completarse en su propia interrupción? Es un autor que está en una intensa crisis sicológica, que está en la cima de su carrera (el discurso de aceptación del premio Inca Garcilaso de la Vega, uno de los más importantes del Perú, fue designado por Arguedas para hacer de prólogo), que puede entablar discusiones con figuras internacionales como Julio Cortázar, y sobre todo como un narrador que existe en la medida en que puede continuar escribiendo: la escritura como fuente de vida. Si se piensa de nuevo en la mención a la capacidad perdida de insuflar la palabra con la "materia de las cosas" que aparecía en las primeras líneas de la novela, cabe recordar que esta capacidad aparece cuando el autor recupera su "tono de vida". Así la escritura y, sobre todo, la capacidad de imprimir en ella la "materia" del mundo, es lo que se constituye como la fuerza vital del narrador de los diarios.

Teniendo en cuenta lo anterior, se podría decir que a través de los diarios se asiste a la extinción de la posibilidad de traducción de las experiencias basadas en la sensibilidad quechua a alguna de las particulares formas de español en Chimbote, posibilidad que constituía la manera en que el narrador de las anteriores obras de Arguedas daba cuenta de una unión directa con el mundo a través del lenguaje (Lee 23). Tal sensibilidad serrana se había constituido sobre la base de la trayectoria de Arguedas, incluso cuando desde 1961 se distanció del género indigenista. El narrador de los diarios, autor ficticio de Los zorros, hace mención también de esa trayectoria basada en la experiencia que ha construido al pertenecer a dos culturas, y menciona igualmente los matices acerca de sus posiciones políticas. Lo interesante es que lo hace considerándose a sí mismo como un parteaguas entre dos épocas del Perú, y sintiéndose incapaz de reconstruirse a sí mismo en la época que comienza: 
Quizá conmigo empieza a cerrarse un ciclo y a abrirse otro en el Perú y lo que él representa: se cierra el de la calandria consoladora, del azote [...]; se abre el de la luz y de la fuerza liberadora invencible del hombre de Vietnam, el de la calandria de fuego, el del dios liberador [...] Despidan en mí un tiempo del Perú. [...] ¿Cómo están las fronteras de alambres de púas, Comandante? (Arguedas, El zorro... 246)

Como estoy seguro que mis facultades y armas de creador, profesor, estudioso e incitador, se han debilitado hasta quedar casi nulas y solo me quedan las que me relegarían a la condición de espectador pasivo e impotente de la formidable lucha que la humanidad esté librando en el Perú y en todas partes, no me sería posible tolerar ese destino. $\mathrm{O}$ actor, como he sido desde que ingresé a la escuela secundaria, hace cuarentaitrés años, o nada. (Arguedas, El zorro... 250)

En estas dos citas se encuentran las concepciones del autor ficticio de la novela respecto del momento histórico que se vivía después de 1968, y de su propio papel en tales circunstancias. De estas citas y lo expuesto anteriormente, se puede concluir que las capacidades que lo hacían un "actor" no pasivo en la sociedad peruana se relacionan directamente con la de insuflar la "materia de las cosas" en su escritura, pero que al estar ahora mermada, no le permitirían vivir ese nuevo tiempo que es revolucionario: nombra a Vietnam, al "Comandante" (seguramente el Che Guevara), y la "formidable lucha" de la humanidad. Encontramos en estas citas una correlación entre el nacimiento de una época revolucionaria y la desaparición del mundo "del azote" y "del arrieraje": el mundo casi feudal de la sierra. De los fragmentos citados se puede entender también cómo Arguedas era consciente de la "refracción" de los conflictos políticos latinoamericanos al interior de los campos literarios peruano y latinoamericano, y de cómo su novela expresaba una toma de posición al respecto.

La sensibilidad serrana y su potencial político se construía a partir de una experiencia de trabajo comunitario muy propio de las comunidades indias de los Andes peruanos. En Los zorros, al acontecer en un medio costero, industrializado y urbano, tal tipo de trabajo no aparece, y tampoco tal sensibilidad serrana, por lo menos de la manera en que lo venía haciendo en las obras anteriores. No hay ningún personaje que de alguna manera exprese directamente tal sensibilidad, como Gabriel o Rendón, y no se puede decir que haya en la novela una 
especial atención en destacar el aspecto de "comunión con el mundo". A pesar de que hay mención a ciertas características humanas destacables de aquellos que vienen de la sierra - como la cooperación entre pares-, no es posible encontrar en la novela un punto de vista crítico serrano que contraste con otras formas de sensibilidad. Personajes como Diego - ese extraño y "alevitado hippi" — o Hilario Caullama, evidentemente reflejan un contacto con la sierra, pero distan de ser protagonistas o de representar el eje de la novela. Por ejemplo, Diego es un personaje con visos fantásticos, mientras Caullama es una figura secundaria. El loco Moncada, que se podría entender como uno de los protagonistas de la novela, y cuyo discurso parece articular de manera particular el mundo de Chimbote, no tiene una sensibilidad marcadamente serrana. Para decirlo sucintamente en palabras de Jean Franco (2002), en Los zorros "the power of the indigenous derives from its territoriality, a territoriality that has sacred configurations" (173): alejada de la sierra, pareciera que la fuerza mágica, y al tiempo emancipatoria de las prácticas comunitarias indias, se disipara. Por otro lado, las relaciones con el trabajo de los personajes de la novela están mediadas por la presencia de Braschi, ese capitalista peruanísimo cuyos tentáculos de poder se extienden por Chimbote casi de manera ubicua, negando así la posibilidad de un tipo de trabajo comunitario. Así, mientras en los diarios el narrador confesaba que su capacidad revolucionaria estaba radicalmente mermada, en los capítulos de Chimbote desaparecían los elementos que en las anteriores novelas de Arguedas se podían considerar revolucionarios o críticos frente a la realidad política del Perú.

De este modo, de la lectura paralela de estos registros narrativos -los diarios y los capítulos sobre Chimbote- surgen significativas relaciones: la novela parecería insinuar una intersección entre la incapacidad del autor y la migración india a la costa, como si la capacidad revolucionaria de aquel desapareciera debido a la transformación de los indios en Chimbote. Esta idea se ve reforzada por el hecho de que el narrador se considere como un parteaguas en la historia del Perú, y relacione el comienzo de una época revolucionaria con el final del mundo serrano: si este desaparece, y con él la posibilidad de una sensibilidad diferente y crítica del mundo contemporáneo, la capacidad revolucionaria del narrador desaparecería también. Es necesario tener en cuenta que esta relación es parte de la ficción de la novela. 
De igual modo, es importante recordar que en 1968 Arguedas visitó Cuba y dictó una conferencia titulada "La literatura peruana", en donde traza una diferencia entre los nuevos narradores peruanos - entre ellos Vargas Llosa - y los que tratan el tema indio: Alegría y Arguedas, quien retoma el tema de las técnicas narrativas como una cuestión de influencia extrajera que trae como consecuencia el "adelgazamiento" de la esencia puramente peruana (Obra... 7, 537). También en dicha conferencia Arguedas menciona una lectura politizada de Los ríos profundos: el levantamiento de los indios de hacienda por motivos religiosos - la intención de matar a la "madre de la peste" - insinuaba su capacidad revolucionaria en estado embrionario, que se potenciaría cuando se cambiaran esas motivaciones irracionales por otras que tuvieran más relación real con el mejoramiento de sus condiciones de vida. Después de su viaje a la isla del caribe, Arguedas publicó una nota referente al trigésimo aniversario de la muerte de César Vallejo, en donde hacía, hasta donde tenemos entendido por vez primera, una mención pública bastante elogiosa de la revolución liderada por Castro $($ Obra... 7, 550). Es, pues, notable su adhesión a la causa cubana, y también su reiteración de las potencialidades revolucionarias de las comunidades indias. Sin embargo, las condiciones políticas del Perú habían cambiado bastante desde la publicación de Los ríos profundos, y desde las tomas de tierras de comienzos de la década. Si los levantamientos liderados por Hugo Blanco se habían constituido como una muestra evidente de las posibilidades políticas de los indios, hacía ya cinco años que Blanco había sido capturado, el gobierno de Belaúnde se había "derechizado" y los procesos guerrilleros habían sido derrotados. Además, las migraciones hacia la costa, a pesar de haber organizaciones barriales que Arguedas admiraba y respetaba, no propiciaron propuestas políticas más concretas. Esta diferencia estructural entre el campo político peruano y el campo político latinoamericano estaría expresada también en la novela.

En este sentido cabe recodar la manera en que Jean Franco pone a Arguedas como autor que de alguna manera hace tambalear las posturas de Casanova revisadas en Sánchez respecto de la mundialización de las letras: "Es Arguedas más actual que Cortázar, ya que su obra ha provocado discusiones sobre transculturación y heterogeneidad, temas candentes en la época de migraciones. Es Arguedas más que el autor de Fantomas y los multinacionales, pese a que este captó el lado negro de la 
globalización" (188). Esta visión, compartida por Moraña, señala que la obra de Arguedas se inscribe precisamente en la defensa de lo nacional como problema y, se podría decir "en contra" de una concepción globalizada o mundializada de la literatura. Lo que hemos visto hasta acá es que esta postura aparece de manera explícita y radical en el debate con Cortázar debido, específicamente, a la escisión del campo literario latinoamericano y, sobre todo, a la tensión que existió - y que Arguedas captó en la novela-, entre las esperanzas revolucionarias latinoamericanas y la imposibilidad de una organización política india o serrana. Tal imposibilidad señalaba la aporía de la nación moderna latinoamericana, y por esto Los zorros puede ser ubicada como una piedra en el zapato en la teoría de Casanova, al contrario de Cortázar o Vargas Llosa, quienes no parecen interesados en reconocer tal aporía, e incluso se inclinarían por esconderla, negarla o menospreciarla, cuando no en tomar partido abiertamente por la desaparición de las comunidades indias.

Así, la esperanza política de Arguedas - y del narrador de los diarios- estaba puesta en la situación internacional: Cuba y Vietnam, pero no existía ninguna expresión de entusiasmo por una posibilidad peruana de emancipación política, como la llegó a haber con Hugo Blanco, o con los primeros momentos del gobierno de Belaúnde. Así que en la novela parecen cruzarse los dos polos de esta contradicción política: la esperanza en la humanidad - especialmente en Latinoamérica-, y la desesperanza al interior del Perú. Las posibilidades políticas de una literatura que conservara la sensibilidad serrana se hacían, para el narrador de Los zorros, cada vez menores en el Perú, mientras que tal sensibilidad era de hecho la que podría aportar revolucionariamente a la causa cubana y latinoamericana. Al mismo tiempo, el polo "realista" al que más fácilmente se podía adherir la trayectoria de Arguedas - basada en la expresión literaria de la sensibilidad serrana- (por la manera en que su capital cultural se había acumulado) se privilegiaba en las discusiones del campo literario latinoamericano.

Teniendo lo anterior presente, es posible ahora observar las implicaciones que tiene el hecho de que Arguedas construyera su novela más "vanguardista" precisamente en el momento en que toda su trayectoria como escritor "realista" podría verse favorecida por el cambio en las condiciones de las políticas culturales en Cuba y en los debates latinoamericanos al respecto. 
Hay que aclarar en primera instancia que Los zorros, aunque más "vanguardista" que las anteriores obras de su autor, tiene un fuerte componente "realista", en cuanto pretender retratar de manera más o menos fiel la vida en Chimbote. En esta medida, lo que hace Arguedas es crear una novela que retoma la posibilidad de aunar nuevamente "vanguardismo" y "realismo" pero, al mismo tiempo, que se muestra a sí misma como una obra incompleta e imposible: Los zorros denuncia así, a través de su propio ejemplo, la crisis insuperable de la permanencia de tal dualidad en el campo literario latinoamericano, pero lo hace expresando la imposibilidad de que en el Perú las masas indias participen políticamente de la transformación de la humanidad que para Arguedas estaba ocurriendo en el mundo, empezando por Cuba y por Vietnam. La novela muestra cómo, sin un sustento político que haga posible la emancipación india, el "realismo" pierde su carga crítica y el "vanguardismo" se convierte en mera experimentación con un lenguaje que ha perdido ya su capacidad de transmitir un contacto "material" con el mundo. Así, la migración india a la costa desaprovecha, en la novela, sus posibles tonos épicos y triunfales - los que se veían por ejemplo en el poema a Tupac Amaru-, y se convierte en una especie de fracaso monumental de la esperanza de ver a esas masas emancipadas. La "transculturación" se entiende, entonces, sin la apertura de una posibilidad política real para los indios, como retórica "vanguardista". De esta manera, Los zorros al mismo tiempo señala la impotencia política india al interior del Perú, mientras adhiere a una posición revolucionaria en el campo literario latinoamericano, siendo este tal vez uno de los dilemas, entre otros tantos, que se expresan en la obra: uno más de los "double binds" que Mabel Moraña (2013) enseña en su libro sobre Arguedas y Vargas Llosa.

Por último, habría que observar la cuestión de la muerte del narrador dentro de la novela misma, y su relación con el suicidio real de Arguedas. La novela toda aparece como el relato de una agonía, de una muerte aplazada constantemente a través de la escritura misma.

Morir era antaño un proceso público y altamente ejemplar en la vida del individuo [...] Pero es ante nada en el moribundo que, no solo el saber y la sabiduría del hombre adquieren una forma transmisible, sino sobre todo su vida vivida, y ese es el material del que nacen las historias. [...] En el origen de lo narrado está esa auto- 
ridad [...] Pero el lector de novelas busca efectivamente, personas en las que pueda efectuar la lectura del "sentido de la vida". Por lo tanto, sea como fuere, debe tener de antemano la certeza de asistir a su muerte. (Benjamin 9-14)

Cito a Benjamin en cuanto encuentro en este texto dos puntos clave: la cuestión de la muerte como un proceso que ha perdido su condición de evento público, y como acontecimiento que brinda sentido y autoridad a las narraciones de quien agoniza. En Los zorros el narrador descubre en su agonía que el sentido de su vida ha sido imprimir al lenguaje una conexión íntima con el mundo, con unas connotaciones revolucionarias. Al perder dicha capacidad, su vida pierde significado, y esa carencia es retratada también en la novela. Es decir que la novela, al mismo tiempo, expone el sentido de la vida del narrador y retrata la pérdida de ese sentido.

Como se decía, el aparente final del mundo serrano se relacionaba directamente con la capacidad de escribir del narrador, de manera que el sentido de su vida solo es posible mientras exista tal mundo. La muerte de narrador es el final del mundo serrano, y así el relato sobre Chimbote serían el testimonio final de ese mundo. Este hecho, sumado a que el autor real también comete suicido, le dan a tal muerte un carácter público notable, haciendo de la novela no solo un relato sobre la muerte, sino también las palabras de un hombre real agonizante, cuyo sentido de la vida también aparecería en tal relato. Es decir que el sentido de la vida del hombre público Arguedas - su trayectoria y el capital cultural que ha acumulado- que se manifestaría en la novela, y que podemos interpretar a partir de las ideas de Benjamin, estaba íntimamente ligado al destino del mundo andino, y que sin la existencia de este no sería posible continuarla. Por otro lado, si pensamos en Arguedas como un personaje casi emblemático de la historia literaria y cultural del Perú, su suicidio y la novela que escribió mientras lo concebía serían una especie de denuncia pública en donde se mostraría cómo la potencia creadora del país perdería parte importantísima de sí misma al desaparecer el mundo andino. Una denuncia, en última instancia, de la ausencia de una posibilidad política india que asegurara la supervivencia de la sensibilidad serrana.

Es acá donde es importante diferenciar la interpretación literaria que se puede hacer de la novela y del significado que le suma el sui- 
cidio real de Arguedas, de la situación real de la persona histórica. Si bien en la novela el Arguedas real intenta confundirse con el personaje ficticio que escribe los diarios y se presenta a sí mismo casi como la encarnación de un periodo histórico que agonizaba, y que su capacidad de escritura mermaba precisamente por esa condición histórica, la verdad era que Arguedas padecía una enfermedad siquiátrica grave desde hacía décadas, y que posiblemente su capacidad de escritura mermara por tal enfermedad, y no tanto por el contexto del país.

Nunca será posible saber hasta qué punto en realidad se cruzaban esos dos ámbitos - el síquico y el histórico—- pero sí es posible afirmar que Los zorros es una ficcionalización poderosa de tal condición personal. Esta diferenciación se evidencia en un texto que Arguedas publicó en 1969, un par de meses antes de suicidarse y con buena parte de la novela ya escrita. Para ese entonces, Belaúnde ya había sido derrocado por un golpe de estado liderado por el general Juan Velasco Alvarado. Este nuevo gobierno militar llevaba casi un año realizando varios cambios que recogían la "esperanza" aplazada sucesivas veces en el trascurso de esa década, adelantando una progresista reforma agraria, entre otros cambios. En tal texto, Arguedas manifestaba un fuerte optimismo en que la población india sería valorada y reconocida. Diría, contrariando el pesimismo de la novela:

Si usted [Velasco] y los oficiales del ejército no temen ni desprecian a la juventud, si no temen y desprecian al pueblo como en su verdadero "cielo interno" les temían y despreciaban Belaúnde y Haya [de la Torre, líder del APRA], usted, el ejército, pueden haber lanzado el Perú al gran vuelo; acaso podamos ver esa flecha lanzada al infinito antes de morir. Puede usted confiar en los indios ${ }^{4}\left(\right.$ Obra... 7, 592) $^{2}$

\section{Conclusiones}

En Los zorros, Arguedas abandona hasta cierto punto el tono "realista" que pretendía esgrimir en sus obras anteriores, justo en el momento histórico en donde tal tono se empezaba a considerar hegemónico. Sin embargo, la novela se presenta a sí misma como un intento fallido por consolidar el "realismo vanguardista": tiene como uno de los temas

4 Cursivas del autor. 
principales su propio fracaso. Los zorros, a través de la voz del narrador en los diarios, muestra la incapacidad de su propio lenguaje para continuar expresando el contacto "material" con el mundo, mientras que en los capítulos sobre Chimbote muestra la incapacidad de la sensibilidad india de sobrevivir en ese nuevo medio costero. La voz de los diarios, al tiempo que celebra las nuevas luchas por la emancipación de los pueblos alrededor del globo —resaltando a Cuba y a Vietnam-, se considera a sí misma incapaz de participar activamente en tales procesos. La voz de los diarios también se construye a sí misma como una especie de encarnación de un momento histórico del Perú que estaba llegando a su final: el de la sierra colonial y cerrada en sí misma. Según esa misma voz, la imposibilidad de conservar la sensibilidad que allí se gestaba y se convertía en la incapacidad de hacer parte de las transformaciones políticas mundiales que se vivían en el momento.

Así, Arguedas en su novela muestra la ausencia de una propuesta política específicamente india en Perú, mientras al mismo tiempo celebra los procesos políticos emancipadores en otros países. En este sentido, cabe pensar que la modesta contribución de este artículo a la comprensión del texto arguediano consiste en darle una ubicación concreta en los debates nacionales y regionales que lo vieron nacer y que, de alguna manera, refuerzan desde otra perspectiva las afirmaciones de Mabel Moraña respecto de las implicaciones sociopolíticas de su lectura de Los zorros.

En efecto, primero la autora afirma, a través de una cita de Lienhard, que: "La continuación de El zorro... no podrá ser literaria sino política: la hará el lector colectivo que crece poco a poco, a lo largo de la novela, para convertirse al final, algo míticamente, en actor de la historia" (149). A través de esta cita, Moraña señala uno de los sentidos de la obra que, a partir de una lectura organizada desde otras estructuras teóricas, es compartida también en este artículo: Los zorros apunta a "la activación del indio como sujeto político y como actor social a partir de una agenda de emancipación colectiva" (149).

\section{Referencias bibliográficas}

Arguedas, José María, Bravo Bresani, Jorge, Escobar, Alberto, Favre, Henri, Matos Mar, José, Oviedo, José Miguel, Quijano, Aníbal y Sebastián Salazar Bondy. ¿He vivido en vano? Mesa redon- 
da sobre Todas las sangres. Lima, Instituto de Estudios Peruanos, 1985.

Arguedas, José María. El zorro de arriba y el zorro de abajo. 1971. Madrid, Fondo de Cultura Económica, 1996. Obra antropológica, Tomo 7. Lima, Ed. Horizonte, 2011.

Benjamin, Walter. "Sobre algunos temas en Baudelaire". Sobre el programa de la filosofía futura y otros ensayos. Barcelona, Planeta, 1986, pp. 9-14

Bourdieu, Pierre. Las reglas del arte. Barcelona, Anagrama, 1995.

. "El campo literario: prerrequisitos críticos y principios de método". Criterios, no. 25-28, 1990, pp. 20-42.

Cornejo Polar, Antonio. Los universos narrativos de José María Arguedas. Buenos Aires, Losada, 1973.

Franco, Jean. The decline and fall of the lettered city: Latin America in the Cold War. Cambridge, Harvard University Press, 2002.

Gilman, Claudia. Entre la pluma y el fusil. Dilemas y debates del escritor revolucionario en América Latina. Buenos Aires, Siglo XXI, 2003.

Lee Penagos, Juan Camilo. "Política en El Sexto de José María Arguedas: sensibilidad serrana, magia y realismo". Perifrasis, Revista de Literatura, Teoría y Crítica, vol. 10, no. 20, 2019, pp. 11-28.

Martínez, Ana María. "Para estudiar campos periféricos: un ensayo sobre las condiciones de utilización fecunda de la teoría del campo de Pierre Bourdieu". Trabajo y Sociedad, vol. 8, no.9, 2007, pp. 1-31.

Moore, Melissa. En la encrucijada: las ciencias sociales y la novela en el Perú. Lima, Fondo editorial Universidad Nacional Mayor de San Marcos, 2003. Recuperado de http://sisbib.unmsm.edu. pe/bibvirtual/libros/literatura/encrucijada/contenido.htm. 29 de mayo de 2017.

Moraña, Mabel. La escritura del límite. Madrid, Iberoamericana, 2010. Arguedas/Vargas Llosa. El demonio feliz y el hablador. Madrid, Frankfurt, Iberoamericana/Vervuert, 2013. Web. 2 de julio de 2020.

Moreiras, Alberto. “The end of magical realism: José María Arguedas's passionate signifier ('El zorro de arriba y el zorro de abajo')". The Journal of Narrative Technique, vol. 27, no. 1, 1997, pp. 84-112. 
Sánchez Prado, Ignacio. Ed. América Latina en la "literatura mundial". Pittsburgh, Universidad de Pittsburgh, 2006.

Sigal, Silvia. Intelectuales y poder en la década del sesenta. Buenos Aires, Puntosur editores. 1991. 